\title{
Impact of Intercore Crosstalk on Achievable Information Rates
}

\author{
Daniel J. Elson $\dagger^{1}$, Benjamin J. Puttnam*, Georg Rademacher*, Ruben S. Luis*, Eric Sillekens $\dagger^{*}$, Lidia Galdino $\dagger^{*}$, Domaniç Lavery†, Yoshinari \\ Awaji*, Naoya Wada* and Polina Bayvel $\dagger$ \\ $\dagger$ Dept. Electronic and Electrical Engineering, Optical Networks Group, UCL, London, UK, \\ 1aniel.elson.13@ucl.ac.uk \\ *NICT, 4-2-1, Nukui-Kitamachi, Koganei, Tokyo, 184-8795, Japan
}

\begin{abstract}
We investigate the impact of intercore crosstalk in a 7-core fiber on signal to noise ratio and achievable information rates for PDM-QPSK, PDM-16QAM, and PDM-64QAM formats. The achievable rate is reduced by $8 \%, 5.4 \%$ and $1.5 \%$, at a crosstalk of $-25.6 \mathrm{~dB} / 100 \mathrm{~km}$ respectively
\end{abstract}

Keywords-Multicore fiber, Cross-Talk, Information Rates

\section{INTRODUCTION}

Space-division-multiplexing (SDM) technologies have been widely proposed as a cost-effective solution to increase the transmission capacity in a single fiber by utilizing multiple cores or spatial-modes [1,2]. Single-mode multi-core fibers (MCFs) offer a migration path into SDM technology in the short term. Such fibers have been shown to support high spectral efficiency modulation formats without the complexity of high-order multiple input-multiple output (MIMO) based receivers [3] needed my multimode fibres. MCFs have been used in long-haul transmission, access, data-center and networking demonstrations.

MCFs with different core layout, core pitch, and cladding refractive index profiles and various strategies for reducing the crosstalk interaction and its impact have been investigated $[4,5]$. It was observed that a cross talk (XT) of less than $-32.2 \mathrm{~dB} / 100 \mathrm{~km}$ can reduce transmission distances by up to $40 \%$ [6]. Since XT can be engineered to any required level, here we investigate how it affects the achievable rate of MCF transmission. We transmit polarisation division multiplex (PDM) 4, 16, 64-ary quadrature amplitude modulation (QAM) signals with varying levels of XT over short and long haul distances and measure the signal to noise ratio (SNR). Information theory answers the question regarding the maximum amount of information that can be reliably transmitted over a communication channel [7]. In this work, an ideal coding scheme is assumed in order to investigate the effect of XT independent of coded modulation. The mutual information (MI) between the transmitted and received symbols represents the largest achievable information rate (AIR). For the ideal coding scheme, MI is correct AIR.

\section{EXPERIMENTAL SETUP}

An experimental strategy was devised to emulate different XT values using the same fiber. The set-up is shown in Fig. 1, and was based on a recirculating transmission loop. The signal laser was a $100 \mathrm{kHz}$ linewidth external cavity laser tuned to $1550.116 \mathrm{~nm}$ and modulated in a dual parallel Mach-Zehnder modulator (DP-IQ-Mod). The modulator was driven by four

U.K. Engineering and Physical Sciences Research Council under Grant EP/J017582/1 (UNLOC) and iCASE award with the BBC independent arbitrary waveform generators (AWGs). Each AWG had an analogue bandwidth of $14 \mathrm{GHz}$ and used a sampling rate of $49 \mathrm{GS} / \mathrm{s}$ to generate pre-equalized PDM4QAM, PDM-16QAM and PDM-64QAM signals at 24.5 GBd with a root-raised cosine pulse shape with a roll-off of 0.01 . After modulation, an EDFA boosted the signal power before transmission over a 7-core MCF. Each recirculation of the loop used the centre core of the $53.7 \mathrm{~km} \mathrm{MCF}$ span.

The loop contained two EDFAs and $30 \mathrm{GHz}$ bandpass filter set by a wavelength selective switch (WSS) to limit ASE. Optical taps and variable optical attenuators (VOAs) were used to set and monitor the launch power into the interfering cores. Intercore XT (IC-XT) was emulated by using dummy channels generated from the loop output after each recirculation. The dummy channels were amplified and decorrelated with fiber patch cords before being re-injected into fiber. Taking the dummy channels from the inside the loop ensured that they had experienced transmission and noise degradation similar to the signal channels, emulating a real MCF link. The signal power was maintained and the launch power of the dummy channels varied to give a range of $\mathrm{XT} / \mathrm{span}$ values of $-45.7 \mathrm{~dB}$ to $-21.2 \mathrm{~dB}$, equivalent to $-43.0 \mathrm{~dB}$ to $-18.5 \mathrm{~dB}$ per $100 \mathrm{~km}$.

Acousto-optic modulators (AOMs) were used to control the recirculation time and the receiver was triggered for the required distance. The receiver path from the loop output contained an EDFA, a polarization scrambler (PS) and a VOA for polarization and power control. Signal detection was performed in a polarization-diverse optical coherent receiver connected to a digital sampling oscilloscope with $31 \mathrm{GHz}$ analog bandwidth operating at $80 \mathrm{GS} / \mathrm{s}$. Offline processing was used to recover the signal, consisting of resampling to 2 samples per symbol, followed by normalization and dispersion compensation. Polarization de-multiplexing was performed using a MIMO structure whose equalizers were 33-tap filters

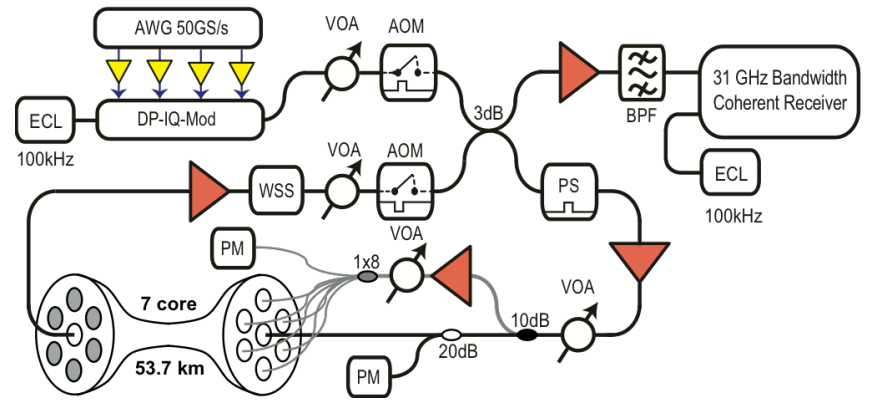

Fig. 1. Experimental Setup to emulate different intercore crosstalk levels 


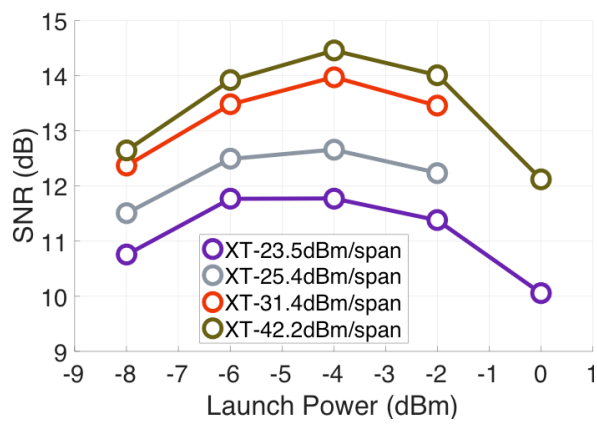

Fig. 2. Signal to noise ratio as a function of launch power at $2148 \mathrm{~km}$ for 16QAM signal with 4 different XT including the highest and lowest

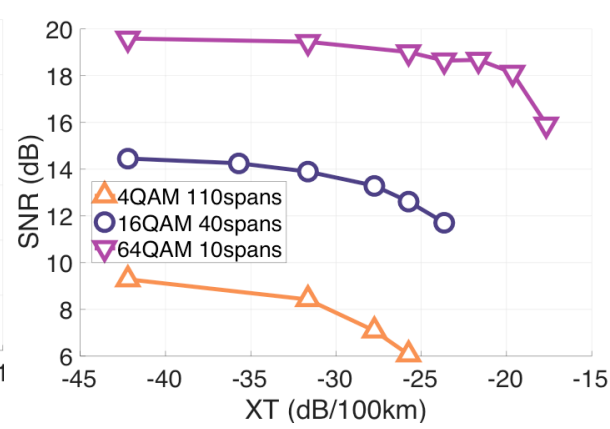

Fig. 4. SNR as a function of XT for 4QAM 16QAM and 64QAM at distances of 5907, 2148 and $537 \mathrm{~km}$ respectively.

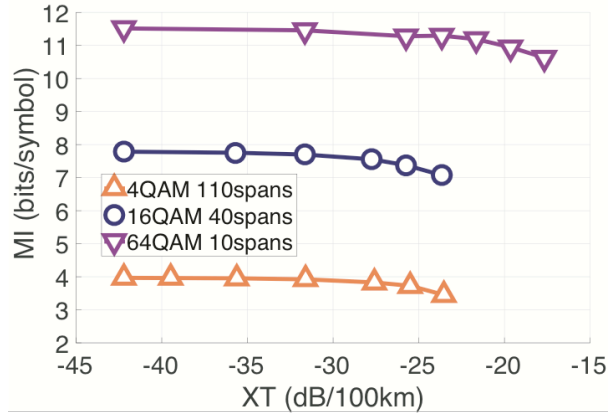

Fig. 3. MI as a function of XT for 4QAM 16QAM and 64QAM at distances of 5907, 2148 and $537 \mathrm{~km}$ respectively updated using a decision-directed least-mean squares algorithm with carrier frequency offset and phase recovery performed in the equalizer loop. Then signal to noise ratio (SNR) measurements were calculated from the average of three traces, (each containing at least 250,000 symbols) using SNR $=\frac{\mathbb{E}\left[|X|^{2}\right]}{\mathbb{E}\left[|Z|^{2}\right]}$ by assuming an additive white Gaussian channel $Y=X+Z$ with transmitted signal $X$, received signal $Y$ and $Z \sim \mathcal{N}\left(0, \sigma^{2}\right)$. The MI was calculated per polarisation on the received symbols and then summed together [8].

\section{RESULTS}

Fig. 2 shows the SNR of a single channel under test carrying PDM-16QAM at 40 spans as a function of launch power for 4 different XT values. At $2148 \mathrm{~km}$ the total accumulated XT for the maximum and minimum values of $\mathrm{XT} / 100 \mathrm{~km}-23.5$ and $-42.2 \mathrm{~dB} / \mathrm{span}$ correspond to -7.48 and $-26.18 \mathrm{~dB}$ respectively. As can be seen the higher the values of XT reduce the SNR in all parts of the curve. The optimum launch power was found to be $-4 \mathrm{dBm}$ for all XT levels. Changing from minimum to maxiumum XT results in a drop in SNR of $2.7 \mathrm{~dB}$ at optimum launch power.

The SNR was then measured at optimum launch power for PDM-4QAM, PDM-16QAM and PDM-64QAM at 110, 40 and 10 spans respectively and is shown in Fig. 3. The back-to-back SNR for 4QAM, 16QAM and 64QAM was measured to be $23.7,24.1$ and $23.9 \mathrm{~dB}$ respectively. The addition of $\mathrm{XT}$ again reduces the SNR in what seems like a very significant way for all tested modulation formats. The lower order modulation formats have their SNR reduce quicker as a function of $\mathrm{XT} / 100 \mathrm{~km}$ due to the greater transmission distances and associated accumulated XT.

In order to understand this in the context of throughput, the effect of XT on AIR is shown in Fig. 4. The back-to-back MI achieved for PDM-4QAM, PDM-16QAM and PDM-64QAM was 4,8 and $11.98 \mathrm{bits} / \mathrm{s} / \mathrm{Hz}$ respectively. At these distances $(110,40$ and 10 spans) a XT level of $-32.2 \mathrm{~dB} / 100 \mathrm{~km}$ (corresponding to the same launch power in all cores [6]), the achievable rate drops by $1.4 \%$ for PDM-4QAM, $1 \%$ for PDM16QAM and $0.2 \%$ for PDM-64QAM. If a loss of $7 \%$ in acheiable rate is permitted $\mathrm{XT}$ can be increased to $-26,-25.2$ and $-19 \mathrm{~dB} / 100 \mathrm{~km}$, for 4QAM, 16QAM and 64QAM respectively. When maximum reach is not a limiting factor in link design, additional XT can be allowed, leading to finer core pitch, which would accomodate more cores in a fixed fibre diameter. This results in more spatial channels to multiplex over giving higher throughput. For PDM-4QAM after a long haul distance of $5,907 \mathrm{~km}$, a XT of $-26.6 \mathrm{~dB} / 100 \mathrm{~km}$ drops the achievable rate by 0.31 bits, a loss of only $7.8 \%$. For PDM$16 \mathrm{QAM}$ at a distance of $2148 \mathrm{~km}$, the rate drops by 0.42 bits (5.4\%) and for PDM-64QAM at drops by 0.17 bits only $1.5 \%$ after $537 \mathrm{~km}$. The increasing loss of AIR with lower modulation formats is a result of the total accumulated XT being higher.

\section{CONCLUSION}

By looking at the effect of XT in terms of achievable information rate the impact on throughput was determined. It is found that achievable rates are not strongly affected by XT. An inter-core crosstalk of $-26.6 \mathrm{~dB} / 100 \mathrm{~km}$ leads to a transmission rate loss of less than $8 \%$.

\section{ACKNOWLEDGMENTS}

Internship Research Fellowship from NICT awarded to Daniel J. Elson is gratefully acknowledged.

\section{REFERENCES}

[1] D. J. Richardson, J. M. Fini, and L. E. Nelson, 'Space-division multiplexing in optical fibres', Nat. Photonics 7 (5), pp. 354-362, Apr. 2013.

[2] G. M. Saridis et al. 'Survey and evaluation of space division multiplexing: From technologies to optical networks', IEEE Commun. Surv. Tutor. 17 (4), pp. 2136-56, Nov. 2015.

[3] B. J. Puttnam et al., ' $2.15 \mathrm{~Pb} / \mathrm{s}$ transmission using a 22 core homogeneous single-mode MCF and wideband optical comb', PDP3-1 ECOC, 2015

[4] A. Sano et al., 'Crosstalk-managed high capacity long haul multicore fiber transmission with propagation-direction interleaving,' J. Lightw. Technol., vol. 32, no. 16, pp. 2771-2779, Aug. 2014.

[5] K. Takenaga et al., 'Reduction of crosstalk by trench-assisted multi-core fiber,' in Proc. IEEE OFC/NFOEC, pp. 1-3, 2011.

[6] B. J. Puttnam, et al., 'Impact of Intercore Crosstalk on the Transmission Distance of QAM Formatsin Multicore Fibers' IEEE Photonics Letters Vol. 8, No. 2, 2016

[7] T. M. Cover \& J.A. Thomas, 'Elements of information theory,' 2nd edition. Wiley, New York (USA); ISBN: 978-0-471-24195-9, 2006.

[8] R. Maher, A. Alvarado, D. Lavery, and P. Bayvel, 'Increasing the information rates of optical communications via coded modulation: a study of transceiver performance,' Sci. Rep. 6, 21278 (2016). 